\title{
Self-Compensation Mechanism in Semi-Insulating CdMnTe Crystals Intended for $\mathrm{X} / \boldsymbol{\gamma}$-Ray Detectors
}

\author{
N.S. Yurtsenyuk, L.A. Kosyachenko, V.M. Sklyarchuk, O.L. Maslyanchuk, \\ O.F. Sklyarchuk and E.V. Grushko
}

Optoelectronics Department, Chernivtsi National University, 58012 Chernivtsi, Ukraine

The electrical properties of single $\mathrm{Cd}_{1-x} \mathrm{Mn}_{x} \mathrm{Te}(x=0.07-0.39)$ crystals with a resistivity of $\approx 10^{8} \Omega \mathrm{cm}$ at $300 \mathrm{~K}$ have been studied. The electrical conductivity is explained in the terms of statistics of electrons and holes in a semiconductor taking into account the compensation process in impurity-defect complexes. The energy of ionization and the degree of compensation levels have been found.

PACS: 72.20.Fr, 72.80.Ey, 78.20.Ci

\section{Introduction}

$\mathrm{Cd}_{1-x} \mathrm{Mn}_{x} \mathrm{Te}$ is a typical diluted magnetic semiconductor known to adopt the zinc-blende structure within the $0<x<0.77$ range. Owing to its unique magnetic and magnetooptic properties [1], $\mathrm{Cd}_{1-x} \mathrm{Mn}_{x}$ Te crystals can be used in many device applications [2] and offer several potential advantages over $\mathrm{Cd}_{1-x} \mathrm{Zn}_{x}$ Te as a material for room-temperature $\mathrm{X}$ - and gamma-ray detectors $[3,4]$. The band gap range $1.7 \mathrm{eV}$ to $2.2 \mathrm{eV}$, required for substantiating an "ideal" radiation detector performance, can be realized with a relatively low $(<50 \%)$ amount of $\mathrm{Mn}$ compared with that of $\mathrm{Zn}$ in $\mathrm{Cd}_{1-x} \mathrm{Zn}_{x}$ Te (up to $70 \%$ ) [4].

In this work we study on mechanism of compensation of $\mathrm{Cd}_{1-x} \mathrm{Mn}_{x}$ Te single crystals with different manganese content. The band gap and its temperature dependence are found from the optical transmission curves. We have succeeded in determining the ionization energy of the energy level responsible on electrical conductivity and the degree of its compensation.

\section{Samples}

$\mathrm{Cd}_{1-x} \mathrm{Mn}_{x}$ Te $p$-type crystals have been grown by the modified melting method in graphite coated quartz ampoules. The MnTe fractions for $\mathrm{Cd}_{1-x} \mathrm{Mn}_{x}$ Te were 0.28 and 0.39 as well as 0.07 for comparison.

Figure 1 shows a correlation of the obtained absorption curves $\alpha(h \nu)$ with a formula that describes the absorption edge for a direct-band semiconductor such as $\mathrm{Cd}_{1-x} \mathrm{Mn}_{x}$ Te [5]:

$$
\alpha=\alpha_{0}\left(h \nu-E_{\mathrm{g}}\right)^{1 / 2},
$$

where $\alpha_{0}$ is the coefficient, which can be considered as independent of photon energy $h \nu$ in a narrow spectral range.

Extrapolation of linear plots of $\alpha^{2}$ vs. $h \nu$ to the intersection with the abscissa axis allows to determine accurately the band gap of the investigated crystals (Table I).

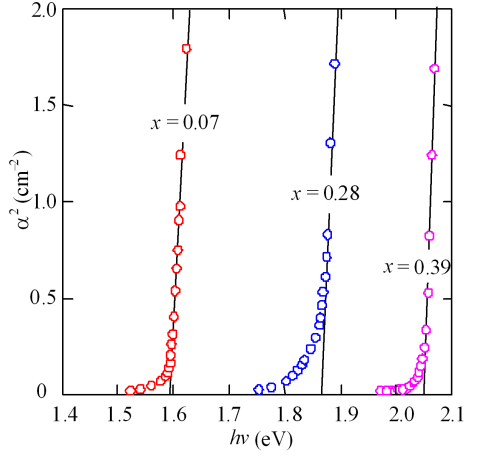

Fig. 1. Absorption curves near the edge of the band gap at $T=300 \mathrm{~K}$ for $\mathrm{Cd}_{1-x} \mathrm{Mn}_{x}$ Te crystals with different content of $\mathrm{Mn}$.

TABLE I

Parameters of $\mathrm{Cd}_{1-x} \mathrm{Mn}_{x}$ Te single crystals under study.

\begin{tabular}{c|c|c|c}
\hline \hline Material & $x$ & $\begin{array}{c}E_{\mathrm{g}}(300 \mathrm{~K}) \\
{[\mathrm{eV}]}\end{array}$ & $\begin{array}{c}\rho \text { at } 300 \mathrm{~K} \\
{[\Omega \mathrm{cm}]}\end{array}$ \\
\hline $\mathrm{Cd}_{1-x} \mathrm{Mn}_{x} \mathrm{Te}$ & 0.07 & 1.59 & $2.5 \times 10^{4}$ \\
$\mathrm{Cd}_{1-x} \mathrm{Mn}_{x} \mathrm{Te}$ & 0.28 & 1.875 & $1.5 \times 10^{8}$ \\
$\mathrm{Cd}_{1-x} \mathrm{Mn}_{x} \mathrm{Te}$ & 0.39 & 2.045 & $7.1 \times 10^{7}$
\end{tabular}

The dependences of $E_{\mathrm{g}}$ on $x$ for $\mathrm{Cd}_{1-x} \mathrm{Mn}_{x}$ Te crystals have been investigated in several works [6-8]. For determining the content of $\mathrm{Mn}$ in the $\mathrm{Cd}_{1-x} \mathrm{Mn}_{x}$ Te single crystals we used the dependence $E_{\mathrm{g}}(x)$ from [7], which gives the best agreement with the $E_{\mathrm{g}}$ value for CdTe at $300 \mathrm{~K}$

$$
E_{\mathrm{g}}=1.47+1.45 x \text {. }
$$

Hence, from the found value of band gap we can determine the $\mathrm{Mn}$ content in the crystals as $x=\left(E_{\mathrm{g}}-\right.$ 1.47)/1.45.

From a practical point of view, the important characteristic required to calculate the conductivity is the tem- 


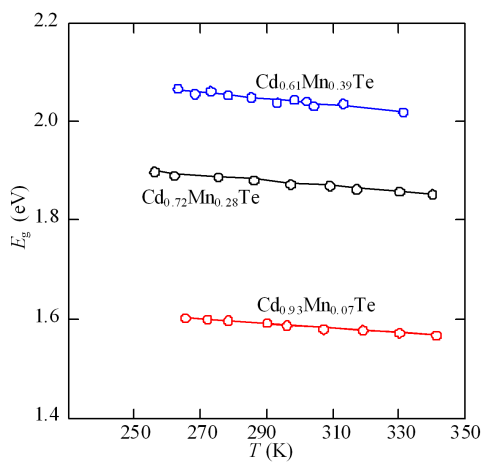

Fig. 2. Temperature dependences of the band gap of $\mathrm{Cd}_{1-x} \mathrm{Mn}_{x} \mathrm{Te}(x=0-0.40)$.

perature dependence of the band gap $E_{\mathrm{g}}(T)$. Figure 2 presents the temperature dependence of the band gaps for crystals with different content of $\mathrm{Mn}$.

As seen, the temperature dependences $E_{\mathrm{g}}(T)$ can be described by function which can be represented as

$$
E_{\mathrm{g}}(T)=E_{\mathrm{g}}(0)-\gamma T,
$$

where $E_{\mathrm{g}}(0)$ is the band gap at $T \rightarrow 0$, and $\gamma$ is its temperature coefficient.

\section{Electrical properties of $\mathrm{Cd}_{1-x} \mathrm{Mn}_{x} \mathrm{Te}$ crystals and their discussion}

Ohmic contacts to $\mathrm{Cd}_{1-x} \mathrm{Mn}_{x}$ Te crystals were made by vacuum evaporation of Ni. Prior the deposition, crystal surface was chemically treated. The contacts provide the linearity of $I-V$ characteristics in a wide range for both polarities of the applied voltage.

Figure 3 represents the temperature dependences of resistivity $\rho$ of $\mathrm{Cd}_{1-x} \mathrm{Mn}_{x}$ Te crystals. As it is seen, the temperature dependences of $\rho$ of all samples in the coordinates $\lg (\rho)$ vs. 1000/T agree with the expected linear functions since the expression for $P$ contains the factor $\exp (\Delta E / k T)$, where $\Delta E$ is the activation energy. The room-temperature resistivity of $\mathrm{Cd}_{0.93} \mathrm{Mn}_{0.07} \mathrm{Te}\left(E_{\mathrm{g}}=\right.$ $1.59 \mathrm{eV})$ is $2.5 \times 10^{4} \Omega \mathrm{cm}$, for $\mathrm{Cd}_{0.72} \mathrm{Mn}_{0.28} \mathrm{Te}\left(E_{\mathrm{g}}=\right.$ $1.875 \mathrm{eV})$ it is much higher and equal to $1.5 \times 10^{8} \Omega \mathrm{cm}$, for the sample with a wider band gap $\mathrm{Cd}_{0.61} \mathrm{Mn}_{0.39} \mathrm{Te}$ $\left(E_{\mathrm{g}}=2.045 \mathrm{eV}\right)$ it is not higher but even somewhat lower. The observed values of $\rho$ for all $\mathrm{Cd}_{1-x} \mathrm{Mn}_{x}$ Te crystals are much lower than the resistivity of samples of these materials with intrinsic conductivity

$$
\rho_{\mathrm{i}}=\frac{1}{q n_{\mathrm{i}} \mu_{\mathrm{n}}+q n_{\mathrm{i}} \mu_{\mathrm{p}}},
$$

where $q$ is the elementary charge, $\mu_{\mathrm{n}}$ and $\mu_{\mathrm{p}}$ are the mobilities of electrons and holes, respectively, $n_{\mathrm{i}}$ is the intrinsic carrier concentration

$$
n_{\mathrm{i}}=\sqrt{N_{\mathrm{c}} N_{\mathrm{v}}} \exp \left(-\frac{E_{\mathrm{g}}}{2 k T}\right),
$$

where $N_{\mathrm{c}}=2\left(m_{\mathrm{n}}^{*} k T / 2 \pi \hbar\right)^{3 / 2}$ and $N_{\mathrm{v}}=2\left(m_{\mathrm{p}}^{*} k T / 2 \pi \hbar\right)^{3 / 2}$

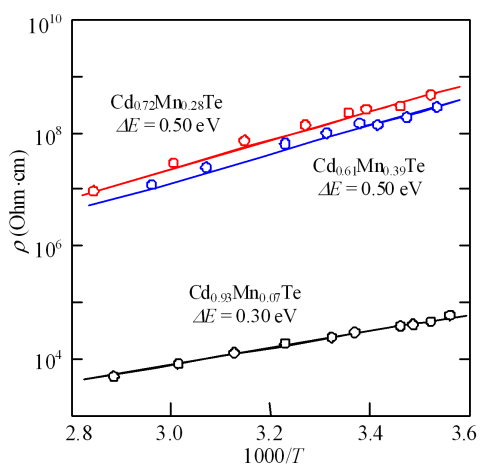

Fig. 3. Temperature dependences of resistivity $\rho$ of $\mathrm{Cd}_{1-x} \mathrm{Mn}_{x} \mathrm{Te}$ single crystals $(x=0.07, x=0.28$, $x=0.39$ ). The activation energies of the conductivity $\Delta E$ are also shown.

are the effective densities of states in the conduction and valence bands, respectively.

We believe that all the samples have an extrinsic (impurity) character of electrical conduction.

As it is known, even high-purity CdTe crystals and, most probably, the $\mathrm{Cd}_{1-x} \mathrm{Mn}_{x}$ Te crystals commonly contain high concentration of residual impurities and intrinsic defects of both donor and acceptor types. Therefore, explaining the above electrical characteristics it is natural to apply compensated semiconductor model. Data on the effective masses of electrons and holes in $\mathrm{Cd}_{1-x} \mathrm{Mn}_{x} \mathrm{Te}$ with different $\mathrm{Mn}$ content are not mentioned in the literature, therefore to estimate we take them the same as for CdTe, i.e. $m_{\mathrm{n}}^{*}=0.11 m_{0}, m_{\mathrm{p}}^{*}=0.35 m_{0}\left(m_{0}\right.$ is the electron mass in vacuum). Again, to make a crude estimate we take $\mu_{\mathrm{n}}=1000 \mathrm{~cm}^{2} /(\mathrm{Vs})$ and $\mu_{\mathrm{p}}=100 \mathrm{~cm}^{2} /(\mathrm{Vs})$ for the resistivity.

Having in mind the hole type of the conductivity of the material, we can accept the Fermi level and electrical conductivity of the material to be determined by acceptor with the ionization energy $E_{\mathrm{a}}$ and the concentration $N_{\mathrm{a}}$. Denoting the concentration of compensating donors by $N_{\mathrm{d}}$, for the electroneutrality equation for a such semiconductor one can write

$$
n+N_{\mathrm{a}}^{-}=p+N_{\mathrm{d}}^{+},
$$

where $N_{\mathrm{a}}^{-}$and $N_{\mathrm{d}}^{+}$are the concentrations of charged donors and acceptors, respectively.

For the wide-band semiconductor under discussion, the Fermi level is located far from the top of valence band and the impurity (defect) concentrations are significant. Therefore we can neglect the concentrations of free carriers $n$ and $p$ in Eq. (7). If the level of compensating donors is shallow enough, they can be considered fully ionized, i.e. we can accept $N_{\mathrm{d}}^{+} \approx N_{\mathrm{d}}$. With these simplifications Eq. (5) reduces to the expression

$$
\frac{N_{\mathrm{a}}}{\exp \left(\frac{E_{\mathrm{a}}-\Delta \mu}{k T}\right)+1}=N_{\mathrm{d}} .
$$


The analytical solution of Eq. (6) has the form

$$
\Delta \mu=E_{\mathrm{a}}-k T \ln \left(\frac{1-\xi}{\xi}\right),
$$

where $\Delta \mu$ is the energy of the Fermi level measured from the top of the valence band, $\xi=N_{\mathrm{d}} / N_{\mathrm{a}}$ is the degree of compensation of acceptors by donors.

Figure 4 shows the temperature dependence of $\Delta \mu$ founded from experimental dependences $\rho(T)$ in Fig. 3 as (using $\left.p=1 / q \rho \mu_{\mathrm{p}}\right)$ :

$$
\Delta \mu=k T \ln \left(\frac{N_{\mathrm{v}}}{p}\right) .
$$

As one can see from Fig. 4, the Fermi level is shifted markedly in the band gap at temperature variations and there are qualitative differences in behavior of $\Delta \mu(T)$ for different samples. For the $\mathrm{Cd}_{1-x} \mathrm{Mn}_{x}$ Te crystals, the energies of the Fermi level $\Delta \mu$ increase with temperature. Analysis shows that the difference between resistivity of the materials determined experimentally and calculated for extrinsic semiconductors is associated with different compensation of the materials.

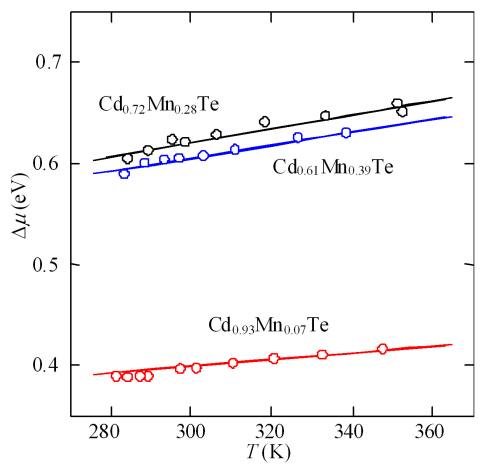

Fig. 4. The temperature dependence of the Fermi level energy $\Delta \mu$ (measured from the top of the valence band) calculated by Eq. (7) (solid lines) and obtained from the curves presented in Fig. 3 using Eq. (8) (circles).

Comparison of calculated and experimental dependences $\Delta \mu(T)$ allows to determine with a rather high accuracy the ionization energy $E_{\mathrm{a}}$ of the level responsible for the electrical conductivity of each sample and the degree of compensation $\xi$ of acceptors by donors. The values of $E_{\mathrm{a}}$ and $\xi$ are summarized in Table II. A high compensation degree $\xi$ of local levels $(0.98-0.9997)$ is typical for the effect of self-compensation impurity centers. It is similar to the observed values of $\xi$ in $\mathrm{CdTe}, \mathrm{Cd}_{1-x} \mathrm{Zn}_{x} \mathrm{Te}$ and other semiconductors $[9,10]$.

\section{Conclusions}

The electrical properties of $\mathrm{Cd}_{1-x} \mathrm{Mn}_{x}$ Te crystals with a resistivity of $10^{4}-10^{8} \Omega \mathrm{cm}$ in the wide range of $\mathrm{Mn}$ content $x=0.07-0.39$ have been studied. The band gaps of the samples $1.59-2.045 \mathrm{eV}$ at $300 \mathrm{~K}$ and their temperature coefficient $(4.4-6.7) \times 10^{-4} \mathrm{eV} / \mathrm{K}$ have been deter-

TABLE II

The electrical parameters of $\mathrm{Cd}_{1-x} \mathrm{Mn}_{x}$ Te single crystals under study at $300 \mathrm{~K}$.

\begin{tabular}{c|c|c|c}
\hline \hline Material & $\Delta E[\mathrm{eV}]$ & $E_{\mathrm{a}}[\mathrm{eV}]$ & $\xi=N_{\mathrm{d}} / N_{\mathrm{a}}$ \\
\hline $\mathrm{Cd}_{0.93} \mathrm{Mn}_{0.07} \mathrm{Te}$ & 0.3 & 0.3 & 0.98 \\
$\mathrm{Cd}_{0.72} \mathrm{Mn}_{0.28} \mathrm{Te}$ & 0.5 & 0.41 & 0.9997 \\
$\mathrm{Cd}_{0.6} \mathrm{Mn}_{0.4} \mathrm{Te}$ & 0.5 & 0.41 & 0.9995
\end{tabular}

mined. It is shown that the conductivity of materials is determined by self-compensation impurities-defects complexes. The ionization energy of such impurities-defects 0.3-0.41 eV and the degree of compensation 0.98-0.9997, which are responsible for the electrical conductivity of the samples under study, have been found.

\section{References}

[1] J.K. Furdyna, J. Appl. Phys. 64, R29 (1988).

[2] R. Triboulet, A. Heurtel, J. Rioux, J. Cryst. Growth 101, 131 (1990).

[3] A. Burger, K. Chattopadhyay, H. Chen, J.-O. Ndap, X. Ma, S. Trivedi, S.-W. Kutcher, R. Chen, R.-D. Rosemeier, J. Cryst. Growth 198/199, 872 (1999).

[4] J.E. Toney, T.E. Schlesinger, R.B. James, Nucl. Instrum. Methods Phys. Res. A 428, 14 (1999).

[5] S. Adachi, Optical Properties of Crystalline and Amorphous Semiconductors: Materials and Fundamental Principles, Kluwer Academic Publishers, Dordrecht 1999.

[6] N. Bottka, J. Stankiewicz, W. Giriat, J. Appl. Phys. 52, 41 (1981).

[7] M. El Amrani, J.P. Lascaray, J. Diouri, Solid State Commun. 45, 351 (1983).

[8] R. Bucker, H.-E. Gumlich, M. Krause, J. Phys. C, Solid State Phys. 18, 661 (1985).

[9] G. Mandel, Phys. Rev. A 134, 1073 (1964).

[10] F.F. Morehead, G. Mandel, Phys. Rev. A 137, 924 (1965). 